\title{
On the almost-principal minors of a symmetric matrix
}

\author{
Shaun M. Fallat* Xavier Martínez-Rivera ${ }^{\dagger}$
}

July 30,2020

\begin{abstract}
The almost-principal rank characteristic sequence (apr-sequence) of an $n \times n$ symmetric matrix is introduced, which is defined to be the string $a_{1} a_{2} \cdots a_{n-1}$, where $a_{k}$ is either $\mathrm{A}, \mathrm{S}$, or $\mathrm{N}$, according as all, some but not all, or none of its almost-principal minors of order $k$ are nonzero. In contrast to the other principal rank characteristic sequences in the literature, the apr-sequence of a matrix does not depend on principal minors. The almost-principal rank of a symmetric matrix $B$, denoted by ap-rank $(B)$, is defined as the size of a largest nonsingular almost-principal submatrix of $B$. A complete characterization of the sequences not containing an A that can be realized as the apr-sequence of a symmetric matrix over a field $\mathbb{F}$ is provided. A necessary condition for a sequence to be the apr-sequence of a symmetric matrix over a field $\mathbb{F}$ is presented. It is shown that if $B \in \mathbb{F}^{n \times n}$ is symmetric and non-diagonal, then $\operatorname{rank}(B)-1 \leq \operatorname{ap}-\operatorname{rank}(B) \leq \operatorname{rank}(B)$, with both bounds being sharp. Moreover, it is shown that if $B$ is symmetric, non-diagonal and singular, and does not contain a zero row, then $\operatorname{rank}(B)=\operatorname{ap}-\operatorname{rank}(B)$.
\end{abstract}

Keywords. Almost-principal minor; almost-principal rank characteristic sequence; enhanced principal rank characteristic sequence; symmetric matrix; rank; ap-rank.

AMS subject classifications. 15B57, 15A15, $15 \mathrm{~A} 03$.

\section{Introduction}

Motivated by work of Brualdi et al. [3] on the principal rank characteristic sequence (prsequence), Butler et al. [5] introduced the enhanced principal rank characteristic sequence (epr-sequence), which they defined as follows: For a given symmetric matrix $B \in \mathbb{F}^{n \times n}$, where $\mathbb{F}$ is a field, the enhanced principal rank characteristic sequence (epr-sequence) of $B$

*Department of Mathematics and Statistics, University of Regina, Regina, Saskatchewan, S4S 0A2, Canada (shaun.fallat@uregina.ca).

${ }^{\dagger}$ Department of Mathematics and Statistics, Auburn University, Auburn, AL 36849, USA (martinez.rivera.xavier@gmail.com). 
is $\operatorname{epr}(B)=\ell_{1} \ell_{2} \cdots \ell_{n}$, where

$$
\ell_{k}= \begin{cases}\mathrm{A} & \text { if all of the principal minors of order } k \text { are nonzero; } \\ \mathrm{S} & \text { if some but not all of the principal minors of order } k \text { are nonzero; } \\ \mathrm{N} & \text { if none of the principal minors of order } k \text { are nonzero (i.e., all are zero), }\end{cases}
$$

where a minor of order $k$ is the determinant of a $k \times k$ submatrix of $B$. (The definition of "principal minor" appears below.) After subsequent work on epr-sequences (see [6, 8, 12, 13]), another sequence, one that refines the epr-sequence, called the signed enhanced principal rank characteristic sequence (sepr-sequence), was introduced by Martínez-Rivera in [14]. Recently, Fallat and Martínez-Rivera [7] extended the definition of the epr-sequence by also taking into consideration the almost-principal minors of the matrix, leading them to a new sequence, which we will define after introducing some terminology: For $B \in \mathbb{F}^{n \times n}$ and $\alpha, \beta \subseteq\{1,2, \ldots, n\}, B[\alpha, \beta]$ will denote the submatrix lying in rows indexed by $\alpha$ and columns indexed by $\beta ; B[\alpha, \beta]$ is a principal submatrix of $B$ if $\alpha=\beta$; the minor $\operatorname{det} B[\alpha, \beta]$ is a principal minor of $B$ if $B[\alpha, \beta]$ is a principal submatrix of $B ; B[\alpha, \beta]$ is an almost-principal submatrix of $B$ if $|\alpha|=|\beta|$ and $|\alpha \cap \beta|=|\alpha|-1$; the minor $\operatorname{det} B[\alpha, \beta]$ is an almost-principal minor of $B$ if $B[\alpha, \beta]$ is an almost-principal submatrix of $B$; the minor $\operatorname{det} B[\alpha, \beta]$ is a quasiprincipal minor of $B$ if $B[\alpha, \beta]$ is a principal or an almost-principal submatrix of $B$; we will say that an $n \times n$ matrix has order $n$; a sequence $t_{1} t_{2} \cdots t_{k}$ from $\{\mathrm{A}, \mathrm{N}, \mathrm{S}\}$ is said to have length $k$. As introduced in [7], for a given symmetric matrix $B \in \mathbb{F}^{n \times n}$, where $\mathbb{F}$ is a field, the quasi principal rank characteristic sequence (qpr-sequence) of $B$ is $\operatorname{qpr}(B)=q_{1} q_{2} \cdots q_{n}$, where

$$
q_{k}= \begin{cases}\mathrm{A} & \text { if all of the quasi-principal minors of order } k \text { are nonzero; } \\ \mathrm{S} & \text { if some but not all of the quasi-principal minors of order } k \text { are nonzero; } \\ \mathrm{N} & \text { if none of the quasi-principal minors of order } k \text { are nonzero (i.e., all are zero) }\end{cases}
$$

A necessary condition for a sequence to be the qpr-sequence of a symmetric matrix over a field $\mathbb{F}$ was found in [7]:

Theorem 1.1. [7, Corollary 2.7] Let $\mathbb{F}$ be a field and $q_{1} q_{2} \cdots q_{n}$ be a sequence from $\{\mathrm{A}, \mathrm{N}, \mathrm{S}\}$. If $q_{1} q_{2} \cdots q_{n}$ is the qpr-sequence of a symmetric matrix $B \in \mathbb{F}^{n \times n}$, then the following statements hold:

(i) $q_{n} \neq \mathrm{S}$.

(ii) Neither NA nor NS is a subsequence of $q_{1} q_{2} \cdots q_{n}$.

The necessary condition in Theorem 1.1 was shown to be sufficient if $\mathbb{F}$ is of characteristic 0 :

Theorem 1.2. [7, Theorem 3.7] Let $\mathbb{F}$ be a field of characteristic 0 . A sequence $q_{1} q_{2} \cdots q_{n}$ from $\{\mathrm{A}, \mathrm{N}, \mathrm{S}\}$ is the qpr-sequence of a symmetric matrix $B \in \mathbb{F}^{n \times n}$ if and only if the following statements hold:

(i) $q_{n} \neq \mathrm{S}$. 
(ii) Neither NA nor NS is a subsequence of $q_{1} q_{2} \cdots q_{n}$.

Theorem 1.2 establishes a contrast between the epr-sequences and qpr-sequences of symmetric matrices, since a complete characterization such as the one in Theorem 1.2 for eprsequences when the field $\mathbb{F}$ is not the field of order 2 is not yet known (see [13]). The absence of such a characterization for epr-sequences is due to the difficulty in understanding epr-sequences containing NA or NS as subsequences. However, in the case of qpr-sequences, this difficulty was overcome, since Theorem 1.1 states that neither NA nor NS can occur as a subsequence of the qpr-sequence of a symmetric matrix [7], regardless of the field; this raises a question:

Question 1.3. Should we attribute the fact that neither NA nor NS can occur as a subsequence of the qpr-sequence of a symmetric matrix entirely to the dependence of qpr-sequences on almost-principal minors?

Question 1.3, together with the applications that almost-principal minors find in numerous areas, which include algebraic geometry, statistics, theoretical physics and matrix theory [7] (see, for example, [2, 9, 10, 11, 15, 16, 17]), is motivation for introducing the almost-principal rank and the almost-principal rank characteristic sequence of a symmetric matrix, which is the focus of this paper:

Definition 1.4. Let $B \in \mathbb{F}^{n \times n}$ be symmetric. The almost-principal rank of $B$, denoted by $\operatorname{ap}-\operatorname{rank}(B)$, is

$$
\operatorname{ap}-\operatorname{rank}(B):=\max \{|\alpha|: \operatorname{det}(B[\alpha, \beta]) \neq 0,|\alpha|=|\beta| \text { and }|\alpha \cap \beta|=|\alpha|-1\}
$$

(where the maximum over the empty set is defined to be 0 ).

We note that, by definition, the ap-rank of a $1 \times 1$ matrix is 0 .

Definition 1.5. For $n \geq 2$, the almost-principal rank characteristic sequence of a symmetric matrix $B \in \mathbb{F}^{n \times n}$ is the sequence (apr-sequence) apr $(B)=a_{1} a_{2} \cdots a_{n-1}$, where

$a_{k}= \begin{cases}\mathrm{A} & \text { if all of the almost-principal minors of order } k \text { are nonzero; } \\ \mathrm{S} & \text { if some but not all of the almost-principal minors of order } k \text { are nonzero; } \\ \mathrm{N} & \text { if none of the almost-principal minors of order } k \text { are nonzero (i.e., all are zero). }\end{cases}$

Some observations highlighting the contrast between apr-sequences and pr-, epr-, seprand qpr-sequences are now in order: Unlike the other sequences, by definition, apr-sequences do not depend on principal minors; moreover, whether or not a matrix is nonsingular is not revealed by its apr-sequence; the apr-sequence of a symmetric matrix $B \in \mathbb{F}^{n \times n}$ has length $n-1$-while the epr-, sepr- and qpr-sequence each has length $n$; furthermore, unlike eprand qpr-sequences, apr-sequences may end with S. Another observation is that the aprsequence of a $1 \times 1$ matrix is simply the empty list (or word), and, therefore, wherever the apr-sequence of an $n \times n$ matrix is involved, we assume that $n \geq 2$.

In the remainder of the present section, some of the terminology we adopted is introduced, known results that are used frequently are listed, and facts about apr-sequences that will 
serve as tools in subsequent sections are established. In Section 2, in particular, we establish a result analogous to Theorem [1.9] below (the NN Theorem for epr-sequences from [5]), as well as a necessary condition for a sequence not containing an A to be the apr-sequence of a symmetric matrix (over an arbitrary field). Section 3 is devoted mostly to apr-sequences not containing an A, which are completely characterized (for an arbitrary field) in Theorem 3.6, and concludes by providing a necessary condition for a sequence (from $\{A, N, S\}$ ) to be the aprsequence of a symmetric matrix (over an arbitrary field). Section 4 is focused on the ap-rank of a symmetric matrix (over an arbitrary field), where it is shown, in particular, that for a symmetric non-diagonal singular matrix $B$ not containing a zero row, $\operatorname{rank}(B)=\operatorname{ap}-\operatorname{rank}(B)$. Section 5 has concluding remarks, including an answer to Question 1.3.

In what follows, unless otherwise stated, $\mathbb{F}$ is used to denote an arbitrary field. Given a vector $x$ of length $n, x[\alpha]$ denotes the subvector of $x$ with entries indexed by $\alpha \subseteq\{1,2, \ldots, n\}$. If the sequence $a_{1} a_{2} \cdots a_{n-1}$ from $\{\mathrm{A}, \mathrm{N}, \mathrm{S}\}$ is the apr-sequence of a symmetric matrix over $\mathbb{F}$, then we will say that the sequence is attainable over $\mathbb{F}$ (or simply that the sequence is attainable, if what is meant is clear from the context). Given a sequence $t_{i_{1}} t_{i_{2}} \cdots t_{i_{k}}$, $\overline{t_{i_{1}} t_{i_{2}} \cdots t_{i_{k}}}$ indicates that the sequence may be repeated as many times as desired (or it may be omitted entirely). The matrices $B$ and $C$ are said to be permutationally similar if there exists a permutation matrix $P$ such that $C=P^{T} B P$. If replacing each of the nonzero entries of a matrix $P$ with a 1 results in a permutation matrix, then we will say that $P$ is a generalized permutation matrix. The zero matrix, identity matrix and all-1s matrix of order $n$ is denoted with $O_{n}, I_{n}$ and $J_{n}$, respectively; moreover, $O_{0}, I_{0}$ and $J_{0}$ are understood to be vacuous. The block diagonal matrix with the matrices $B$ and $C$ on the diagonal (in that order) is denoted by $B \oplus C$.

\subsection{Known results}

In this section, known results that are used frequently are listed, of which some have been assigned abbreviated nomenclature. We start with a well-known fact (see [1], for example), which states that the rank of a symmetric matrix $B$ is equal to the order of a largest nonsingular principal submatrix of $B$; because of this, we will call the rank of a symmetric matrix principal.

Theorem 1.6. [1, Theorem 1.1] Let $B \in \mathbb{F}^{n \times n}$ be symmetric. Then $\operatorname{rank}(B)=\max \{|\gamma|$ : $\operatorname{det}(B[\gamma]) \neq 0\}$.

For a given matrix $B$ having a nonsingular principal submatrix $B[\gamma]$, we denote by $B / B[\gamma]$ the Schur complement of $B[\gamma]$ in $B$ (see [18]). The following result is also a well-known fact (see [4]).

Theorem 1.7. (Schur Complement Theorem.) Let $B \in \mathbb{F}^{n \times n}$ be symmetric with $\operatorname{rank}(B)=$ $r$. Let $B[\gamma]$ be a nonsingular principal submatrix of $B$ with $|\gamma|=k \leq r$, and let $C=B / B[\gamma]$. Then the following statements hold:

(i) $C$ is an $(n-k) \times(n-k)$ symmetric matrix. 
(ii) Assuming the indexing of $C$ is inherited from $B$, any minor of $C$ is given by

$$
\operatorname{det} C[\alpha, \beta]=\operatorname{det} B[\alpha \cup \gamma, \beta \cup \gamma] / \operatorname{det} B[\gamma] \text {. }
$$

(iii) $\operatorname{rank}(C)=r-k$.

Some necessary results about epr-sequences are listed now. The following theorem, which appears in [5], follows readily from Jacobi's determinantal identity.

Theorem 1.8. [5, Theorem 2.4] (Inverse Theorem for epr-Sequences.) Let $B \in \mathbb{F}^{n \times n}$ be symmetric and nonsingular. If $\operatorname{epr}(B)=\ell_{1} \ell_{2} \cdots \ell_{n-1} \mathrm{~A}$, then $\operatorname{epr}\left(B^{-1}\right)=\ell_{n-1} \ell_{n-2} \cdots \ell_{1} \mathrm{~A}$.

Theorem 1.9. [5, Theorem 2.3] (NN Theorem for epr-Sequences.) Let $B \in \mathbb{F}^{n \times n}$ be symmetric. Suppose that $\operatorname{epr}(B)=\ell_{1} \ell_{2} \cdots \ell_{n}$ and $\ell_{k}=\ell_{k+1}=\mathrm{N}$ for some $k$. Then $\ell_{j}=\mathrm{N}$ for all $j \geq k$.

We now state some facts about qpr-sequences.

Observation 1.10. [7, Observation 2.1] Let $B \in \mathbb{F}^{n \times n}$ be symmetric. Then $\operatorname{rank}(B)$ is equal to the index of the last $\mathrm{A}$ or $\mathrm{S}$ in $\operatorname{qpr}(B)$.

Since the rank of a symmetric matrix is principal, it is not hard to show that a statement analogous to Theorem 1.9 must hold for qpr-sequences; however, something stronger does hold: The next result from [7] shows that the presence of a single $\mathrm{N}$ in the qpr-sequence of a symmetric matrix $B \in \mathbb{F}^{n \times n}$ implies that the sequence has Ns from that point forward. This result is of particular relevance later, when we show that an analogous statement does not hold for apr-sequences.

Theorem 1.11. [7, Theorem 2.6] Let $B \in \mathbb{F}^{n \times n}$ be symmetric. Suppose that $\operatorname{qpr}(B)=$ $q_{1} q_{2} \cdots q_{n}$ and $q_{k}=\mathrm{N}$ for some $k$. Then $q_{j}=\mathrm{N}$ for all $j \geq k$.

We conclude this section with a lemma that is used repeatedly, which is immediate from the fact that appending a row and column to a matrix of rank $r$ results in a matrix whose rank is at most $r+2$.

Lemma 1.12. Let $B \in \mathbb{F}^{n \times n}$ be nonsingular. Then the rank of any $(n-1) \times(n-1)$ submatrix of $B$ is at least $n-2$.

\subsection{Tools for apr-sequences}

Some results that will serve as tools to establish results in subsequent sections are provided in this section. The first is an immediate consequence of the Schur Complement Theorem, and it is, therefore, stated as a corollary.

Corollary 1.13. (Schur Complement Corollary.) Let $B \in \mathbb{F}^{n \times n}$ be symmetric, apr $(B)=$ $a_{1} a_{2} \cdots a_{n-1}$ and $B[\gamma]$ a nonsingular principal submatrix of $B$, with $|\gamma|=k$. Let $C=B / B[\gamma]$ and $\operatorname{apr}(C)=a_{1}^{\prime} a_{2}^{\prime} \cdots a_{n-k-1}^{\prime}$. Then, for $j=1, \ldots, n-k-1, a_{j}^{\prime}=a_{j+k}$ if $a_{j+k} \in\{\mathrm{A}, \mathrm{N}\}$. 
A result analogous to the Inverse Theorem for epr-Sequences can be established for aprsequences, by applying Jacobi's determinantal identity:

Theorem 1.14. (Inverse Theorem.) Let $B \in \mathbb{F}^{n \times n}$ be symmetric and nonsingular. If $\operatorname{apr}(B)=a_{1} a_{2} \cdots a_{n-1}$, then $\operatorname{apr}\left(B^{-1}\right)=a_{n-1} a_{n-2} \cdots a_{1}$.

Appending a zero row and a zero column to a matrix is a useful operation, since we can easily determine the apr-sequence of the resulting matrix if we have the apr-sequence of the original matrix, which leads us to the next observation, that is useful when dealing with sequences that do not contain an A.

Observation 1.15. Let $B \in \mathbb{F}^{n \times n}$ be symmetric, apr $(B)=a_{1} a_{2} \cdots a_{n-1}$ and $B^{\prime}=B \oplus O_{1}$. Then $\operatorname{apr}\left(B^{\prime}\right)=a_{1}^{\prime} a_{2}^{\prime} \cdots a_{n-1}^{\prime} \mathrm{N}$, with $a_{j}^{\prime}=a_{j}$ if $a_{j}=\mathrm{N}$, and with $a_{j}^{\prime}=\mathrm{S}$ if $a_{j} \neq \mathrm{N}$, for all $j \in\{1,2, \ldots, n-1\}$.

Another useful tool for working with apr-sequences is the following fact, which is analogous to [5, Theorem 2.6] (Inheritance Theorem for epr-sequences).

Theorem 1.16. (Inheritance Theorem.) Let $B \in \mathbb{F}^{n \times n}$ be symmetric, $m \leq n$, and $1 \leq k \leq$ $m-1$.

1. If $[\operatorname{apr}(B)]_{k}=\mathrm{N}$, then $[\operatorname{apr}(C)]_{k}=\mathrm{N}$ for all $m \times m$ principal submatrices $C$.

2. If $[\operatorname{apr}(B)]_{k}=\mathrm{A}$, then $[\operatorname{apr}(C)]_{k}=\mathrm{A}$ for all $m \times m$ principal submatrices $C$.

3. If $m \geq 6$ and $k \leq m-5$ and $[\operatorname{apr}(B)]_{k}=\mathrm{S}$, then there exists an $m \times m$ principal submatrix $C_{S}$ such that $\left[\operatorname{apr}\left(C_{S}\right)\right]_{k}=\mathrm{S}$.

Proof. Statements (1) and (2) follow from the fact that an almost-principal submatrix of a principal submatrix of $B$ is also an almost-principal submatrix of $B$.

We now establish the final statement. Suppose that $m \geq 6$ and $k \leq m-5$ and $[\operatorname{apr}(B)]_{k}=\mathrm{S}$. Let $p_{1}, p_{2}, \ldots, p_{k-1}, q_{1}, q_{2}, \ldots, q_{k-1}, i, j, r, s \in\{1,2, \ldots, n\}$ be indices such that the following are almost-principal submatrices of order $k$ :

$B\left[\left\{p_{1}, p_{2}, \ldots, p_{k-1}, i\right\},\left\{p_{1}, p_{2}, \ldots, p_{k-1}, j\right\}\right]$ and $B\left[\left\{q_{1}, q_{2}, \ldots, q_{k-1}, r\right\},\left\{q_{1}, q_{2}, \ldots, q_{k-1}, s\right\}\right]$;

moreover, assume that the former submatrix is nonsingular and the latter is singular. Without loss of generality, we may assume that any common indices between the lists $p_{1}, p_{2}, \ldots, p_{k-1}$ and $q_{1}, q_{2}, \ldots, q_{k-1}$ occur in the same position in each list; moreover, we may assume that these common indices (if any) appear consecutively at the beginning of each list. If

$$
\left|\left\{q_{1}, q_{2}, \ldots, q_{k-1}\right\} \cap\{i, j\}\right|=2,
$$

then, without loss of generality, assume that $\left\{q_{k-2}, q_{k-1}\right\}=\{i, j\}$. If

$$
\left|\left\{q_{1}, q_{2}, \ldots, q_{k-1}\right\} \cap\{i, j\}\right|=1
$$


then, without loss of generality, assume that $q_{k-1} \in\{i, j\}$. Consider the following list of almost-principal submatrices of order $k$ :

$$
\begin{gathered}
B\left[\left\{p_{1}, p_{2}, p_{3}, \ldots, p_{k-3}, p_{k-2}, p_{k-1}, i\right\},\left\{p_{1}, p_{2}, p_{3}, \ldots, p_{k-3}, p_{k-2}, p_{k-1}, j\right\}\right], \\
B\left[\left\{q_{1}, p_{2}, p_{3}, \ldots, p_{k-3}, p_{k-2}, p_{k-1}, i\right\},\left\{q_{1}, p_{2}, p_{3}, \ldots, p_{k-3}, p_{k-2}, p_{k-1}, j\right\}\right], \\
B\left[\left\{q_{1}, q_{2}, p_{3}, \ldots, p_{k-3}, p_{k-2}, p_{k-1}, i\right\},\left\{q_{1}, q_{2}, p_{3}, \ldots, p_{k-3}, p_{k-2}, p_{k-1}, j\right\}\right], \\
B\left[\left\{q_{1}, q_{2}, q_{3}, \ldots, p_{k-3}, p_{k-2}, p_{k-1}, i\right\},\left\{q_{1}, q_{2}, q_{3}, \ldots, p_{k-3}, p_{k-2}, p_{k-1}, j\right\}\right], \\
\cdots \\
\left.B\left[q_{1}, q_{2}, q_{3}, \ldots, q_{k-3}, p_{k-2}, p_{k-1}, i\right\},\left\{q_{1}, q_{2}, q_{3}, \ldots, q_{k-3}, p_{k-2}, p_{k-1}, j\right\}\right], \\
\left.B\left[q_{1}, q_{2}, q_{3}, \ldots, q_{k-3}, q_{k-2}, q_{k-1}, r\right\},\left\{q_{1}, q_{2}, q_{3}, \ldots, q_{k-3}, q_{k-2}, q_{k-1}, s\right\}\right] .
\end{gathered}
$$

Since the first submatrix in the above list is nonsingular and the last is singular, this list of submatrices must contain a nonsingular submatrix and a singular submatrix appearing consecutively, say, $B[\alpha, \beta]$ and $B[\gamma, \mu]$. Note that $|\alpha \cup \beta \cup \gamma \cup \mu| \leq k+5 \leq m$. Then by letting $C_{S}$ be an $m \times m$ principal submatrix of $B$ containing $B[\alpha \cup \beta \cup \gamma \cup \mu]$, the desired conclusion follows.

\section{The almost-principal rank characteristic sequence}

We begin with a simple but useful observation.

Observation 2.1. Let $B \in \mathbb{F}^{n \times n}$ be symmetric. Suppose that $\operatorname{apr}(B)=a_{1} a_{2} \cdots a_{n-1}$ and $a_{1}=\mathrm{N}$. Then $B$ is a diagonal matrix and $a_{j}=\mathrm{N}$ for all $j \geq 1$.

Proposition 2.2. Let $B \in \mathbb{F}^{n \times n}$ be symmetric. Suppose that $\operatorname{apr}(B)=\mathrm{A} a_{2} a_{3} \cdots a_{n-2} \mathrm{~N}$ or $\operatorname{apr}(B)=\mathrm{S}_{2} a_{3} \cdots a_{n-2} \mathrm{~N}$. Then $B$ is singular.

Proof. Since $\operatorname{apr}(B)$ does not begin with $\mathrm{N}, B$ is not a diagonal matrix. If $B$ was nonsingular, then, since each of its almost-principal minors of order $n-1$ is zero, $B^{-1}$ is a diagonal matrix, which would imply that $B$ itself is a diagonal matrix, leading to a contradiction.

The NN Theorem for epr-sequences states that if the epr-sequence of a symmetric matrix $B \in \mathbb{F}^{n \times n}$ contains two consecutive Ns, then it must contain Ns from that point forward; the same statement holds for apr-sequences:

Theorem 2.3. (NN Theorem.) Let $B \in \mathbb{F}^{n \times n}$ be symmetric. Suppose that apr $(B)=$ $a_{1} a_{2} \cdots a_{n-1}$ and $a_{k}=a_{k+1}=\mathrm{N}$ for some $k$. Then $a_{j}=\mathrm{N}$ for all $j \geq k$.

Proof. If $k=n-2$, then there is nothing to prove; thus, assume that $k \leq n-3$. It suffices to show that $a_{k+2}=\mathrm{N}$. Suppose to the contrary that $a_{k+2} \neq \mathrm{N}$. Let $B[\alpha \cup\{i\}, \alpha \cup\{j\}]$ be a nonsingular almost-principal submatrix of $B$ with $|\alpha|=k+1$ (note that $i \neq j$ ). We now show that $B$ has a nonsingular $k \times k$ principal submatrix contained in the $(k+1) \times(k+1)$ submatrix $B[\alpha]$. There are two cases:

Case 1: $B[\alpha]$ is nonsingular.

Since $a_{k}=\mathrm{N}$, the Inheritance Theorem implies that every almost-principal minor of $B[\alpha]$ of order $k$ is zero. Then, as $B[\alpha]$ is a $(k+1) \times(k+1)$ nonsingular matrix, its inverse is a 
diagonal matrix, which implies that $B[\alpha]$ is a (nonsingular) diagonal matrix. It now follows immediately that $B[\alpha]$ contains a nonsingular $k \times k$ principal submatrix, as desired.

Case 2: $B[\alpha]$ is singular.

Since $B[\alpha \cup\{i\}, \alpha \cup\{j\}]$ is a nonsingular $(k+2) \times(k+2)$ matrix, Lemma 1.12 implies that $\operatorname{rank}(B[\alpha]) \geq(k+2)-2=k$. Then, as $B[\alpha]$ is $(k+1) \times(k+1)$ singular matrix, $\operatorname{rank}(B[\alpha])=k$. Since the rank of a symmetric matrix is principal, $B[\alpha]$ contains a nonsingular $k \times k$ principal submatrix, as desired.

Let $B[\gamma]$ be a nonsingular $k \times k$ principal submatrix (of $B[\alpha]$ ) with $\gamma \subseteq \alpha$ and $|\gamma|=k$. Then, as $|\gamma|=|\alpha|-1, \alpha=\gamma \cup\{p\}$ for some $p$ (note that $p \neq i, j$ ). Let $C=B / B[\gamma]$ and assume that $C$ inherits the indexing from $B$. Observe that $C$ is an $(n-k) \times(n-k)$ matrix (by the Schur Complement Theorem), and that $n-k \geq 3$. Suppose that $\operatorname{apr}(C)=a_{1}^{\prime} a_{2}^{\prime} \cdots a_{n-k-1}^{\prime}$ Then, as $a_{k+1}=\mathrm{N}$, the Schur Complement Corollary implies that $a_{1}^{\prime}=\mathrm{N}$. It follows from Observation 2.1 that $\operatorname{apr}(C)=\mathrm{NN} \overline{\mathrm{N}}$. Hence, $\operatorname{det} C[\{p, i\},\{p, j\}]=0$. However, by the Schur Complement Theorem,

$$
\begin{aligned}
\operatorname{det} C[\{p, i\},\{p, j\}] & =\frac{\operatorname{det} B[\gamma \cup\{p, i\}, \gamma \cup\{p, j\}]}{\operatorname{det} B[\gamma]} \\
& =\frac{\operatorname{det} B[\alpha \cup\{i\}, \alpha \cup\{j\}]}{\operatorname{det} B[\gamma]} \\
& \neq 0 .
\end{aligned}
$$

Hence, we have a contradiction.

Now that we have the NN Theorem (for apr-sequences), a question arises: Does a statement analogous to Theorem 1.11 hold for apr-sequences? It does not:

Example 2.4. For the matrix

$$
B=\left[\begin{array}{llll}
0 & 1 & 0 & 0 \\
1 & 0 & 0 & 0 \\
0 & 0 & 0 & 1 \\
0 & 0 & 1 & 0
\end{array}\right]
$$

$\operatorname{apr}(B)=$ SNS.

The next result is a corollary to the NN Theorem.

Corollary 2.5. Let $B \in \mathbb{F}^{n \times n}$ be symmetric. Suppose that $\operatorname{apr}(B)$ contains NN. If $\operatorname{apr}(B) \neq$

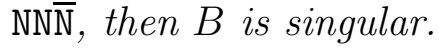

Proof. We will establish the contrapositive. Suppose that $B$ is nonsingular. Let $\operatorname{apr}(B)=$ $a_{1} a_{2} \cdots a_{n-1}$, and suppose that $a_{k} a_{k+1}=$ NN for some $k$. By the NN Theorem, $a_{j}=\mathrm{N}$ for all $j \geq$ $k$. Hence, $\operatorname{apr}(B)=a_{1} a_{2} \cdots a_{k-1} \mathrm{NN} \overline{\mathrm{N}}$. By the Inverse Theorem, $\operatorname{apr}\left(B^{-1}\right)=\overline{\mathrm{N} N N} a_{k-1} \cdots a_{2} a_{1}$. Then, by the NN Theorem, $a_{j}=\mathrm{N}$ for all $j \leq k-1$, implying that $\operatorname{apr}(B)=\mathrm{NN} \overline{\mathrm{N}}$, as desired.

We now show that NA does not occur as a subsequence of the apr-sequence of a symmetric matrix $B \in \mathbb{F}^{n \times n}$. But, to do so, we need a lemma: 
Lemma 2.6. Let $B \in \mathbb{F}^{n \times n}$ be symmetric. Suppose that $\operatorname{apr}(B)=a_{1} \mathrm{~N} a_{3} \cdots a_{n-1}$ and $\operatorname{epr}(B)=\mathrm{N}_{2} \ell_{3} \cdots \ell_{n}$. Then $\operatorname{apr}(B)$ does not contain $\mathrm{A}$.

Proof. If $B=O_{n}$, then the desired conclusion follows by noting that $\operatorname{apr}(B)=\mathrm{NN} \overline{\mathrm{N}}$. Suppose that $B \neq O_{n}$, and let $B=\left[b_{i j}\right]$. By hypothesis, $b_{i i}=0$ for all $i$, implying that $B$ must contain a nonzero off-diagonal entry. Without loss of generality, we may assume that $b_{12} \neq$ 0 . Since every order-2 almost-principal minor of $B$ is zero, $\operatorname{det} B[\{1,2\},\{1, j\}]=0$ and $\operatorname{det} B[\{1,2\},\{2, j\}]=0$ for all $3 \leq j \leq n$. Then, as $\operatorname{det} B[\{1,2\},\{1, j\}]=-b_{1 j} b_{21}=-b_{1 j} b_{12}$ and $\operatorname{det} B[\{1,2\},\{2, j\}]=b_{12} b_{2 j}, b_{1 j}=b_{2 j}=0$ for all $3 \leq j \leq n$. Since $B$ is symmetric, $b_{j 1}=b_{j 2}=0$ for all $3 \leq j \leq n$, implying that $B$ is a block-diagonal matrix with a $2 \times 2$ block. It follows that $a_{1} \neq \mathrm{A}$. Now, note that, for all $k \in\{2,3, \ldots, n-1\}$, the $k \times k$ almost-principal submatrix $B[[k],[k+1] \backslash\{2\}]$ (where $[p]:=\{1,2, \ldots, p\}$ ) is singular, since its first row is zero, as $b_{11}=0$. Hence, $a_{k} \neq \mathrm{A}$ for all $k \geq 2$.

Theorem 2.7. The sequence NA does not occur as a subsequence of the apr-sequence of a symmetric matrix over a field $\mathbb{F}$.

Proof. Let $B \in \mathbb{F}^{n \times n}$ be symmetric, $\operatorname{apr}(B)=a_{1} a_{2} \cdots a_{n-1}$ and $\operatorname{epr}(B)=\ell_{1} \ell_{2} \cdots \ell_{n}$. Suppose to the contrary that $a_{k} a_{k+1}=$ NA for some $k$. By Observation 2.1, $k \geq 2$. We now show that $\ell_{k-1}=\mathrm{N}$. Suppose to the contrary that $\ell_{k-1} \neq \mathrm{N}$. Let $B[\gamma]$ be nonsingular with $|\gamma|=k-1$. By the Schur Complement Corollary, $\operatorname{apr}(B / B[\gamma])=\mathrm{NA} \cdots$, which contradicts Observation 2.1. Hence, $\ell_{k-1}=\mathrm{N}$. By Lemma 2.6, $k-1 \geq 2$. Since $a_{k+1}=\mathrm{A}, \operatorname{rank}(B) \geq k+1$. Then, as the rank of $B$ is principal, the NN Theorem for epr-Sequences implies that $\ell_{k-2} \neq N$. Let $B[\mu]$ be nonsingular with $|\mu|=k-2$. Since $a_{k} a_{k+1}=N A$, the Schur Complement Corollary implies that $\operatorname{apr}(B / B[\mu])=$ YNA $\cdots$ for some $\mathrm{Y} \in\{\mathrm{A}, \mathrm{N}, \mathrm{S}\}$. Since $\ell_{k-1}=\mathrm{N}$, the Schur Complement Theorem implies that $\operatorname{epr}(B / B[\mu])=\mathrm{N} \cdots$, which contradicts Lemma 2.6.

The fact that an analogous version of Theorem 1.11 does not hold in general for aprsequences raises a natural question: What restrictions (if any) can be added to the hypothesis of Theorem 1.11 in order to have its conclusion hold for apr-sequences? Requiring the aprsequence to contain $\mathrm{A}$ as a subsequence is one such restriction:

Theorem 2.8. Let $B \in \mathbb{F}^{n \times n}$ be symmetric. Suppose that $\operatorname{apr}(B)=a_{1} a_{2} \cdots a_{n-1}$ and $a_{k}=\mathrm{A}$ for some $k$. Then neither NA nor NS is a subsequence of $\operatorname{apr}(B)$. Equivalently, if $a_{t}=\mathrm{N}$ for some $t$, then $a_{j}=\mathrm{N}$ for all $j \geq t$.

Proof. By Theorem 2.7, $\operatorname{apr}(B)$ does not contain NA. Suppose to the contrary that $a_{p} a_{p+1}=$ NS for some $p$. Obviously, $p \neq k$ and $p \neq k-1$. Thus, $p \leq k-2$ or $p \geq k+1$. Let $\operatorname{epr}(B)=\ell_{1} \ell_{2} \cdots \ell_{n}$. We now examine all possibilities in two cases.

Case 1: $p \leq k-2$.

By Observation 2.1, $p \geq 2$. We now show that $\ell_{p-1}=\mathrm{N}$. If $\ell_{p-1} \neq \mathrm{N}$, then $B$ has a nonsingular $(p-1) \times(p-1)$ principal submatrix, say, $B[\gamma]$, which would imply that $\operatorname{apr}(B / B[\gamma])=$ $\mathrm{N} \cdots \mathrm{A} \cdots$ (by the Schur Complement Corollary), contradicting Observation 2.1. Hence, $\ell_{p-1}=\mathrm{N}$. By Lemma 2.6, $p \geq 3$. Since $a_{k}=\mathrm{A}, \operatorname{rank}(B) \geq k>p$. Then, as $\ell_{p-1}=\mathrm{N}$ and 
the rank of $B$ is principal, $\ell_{p-2} \neq \mathrm{N}$ (see the NN Theorem for epr-Sequences). Let $B[\mu]$ be a nonsingular $(p-2) \times(p-2)$ (principal) submatrix. Then, by the Schur Complement Corollary and Schur Complement Theorem, $\operatorname{apr}(B / B[\mu])=\mathrm{XN} \cdots \mathrm{A} \cdots$ and $\operatorname{epr}(B / B[\mu])=\mathrm{N} \cdots$, for some $\mathrm{X} \in\{\mathrm{A}, \mathrm{N}, \mathrm{S}\}$, contradicting Lemma 2.6.

Case 2: $p \geq k+1$.

Since $a_{p+1}=\mathrm{S}, \operatorname{rank}(B) \geq p+1$. We proceed by considering two cases.

Subcase A: $B$ contains a nonsingular $(p+1) \times(p+1)$ principal submatrix.

Let $B[\alpha]$ be nonsingular with $|\alpha|=p+1$. By the Inheritance Theorem, $\operatorname{apr}(B[\alpha])=$ $\cdots \mathrm{A} \cdots \mathrm{N}$. Then, by the Inverse Theorem, $\operatorname{apr}\left((B[\alpha])^{-1}\right)=\mathrm{N} \cdots \mathrm{A} \cdots$, which contradicts Observation 2.1.

Subcase B: $B$ does not contain a nonsingular $(p+1) \times(p+1)$ principal submatrix.

Clearly, $\ell_{p+1}=\mathrm{N}$. Since $\operatorname{rank}(B) \geq p+1$, and because the rank of $B$ is principal, the NN Theorem for epr-Sequences implies that $B$ contains a nonsingular $(p+2) \times(p+2)$ principal submatrix, say, $C$. Let $\operatorname{apr}(C)=a_{1}^{\prime} a_{2}^{\prime} \cdots a_{p+1}^{\prime}$ and $\operatorname{epr}(C)=\ell_{1}^{\prime} \ell_{2}^{\prime} \cdots \ell_{p+1}^{\prime} \mathrm{A}$. By the Inheritance Theorem, $a_{k}^{\prime}=\mathrm{A}$ and $a_{p}^{\prime}=\mathrm{N}$. Since every principal submatrix of $C$ is also a principal submatrix of $B, \ell_{p+1}^{\prime}=\mathrm{N}$. Thus far, we have that $\operatorname{apr}(C)=a_{1}^{\prime} a_{2}^{\prime} \cdots a_{k-1}^{\prime} \mathrm{A} \cdots \mathrm{N} a_{p+1}^{\prime}$ and $\operatorname{epr}(C)=\ell_{1}^{\prime} \ell_{2}^{\prime} \cdots \ell_{p}^{\prime} \mathrm{NA}$. By the Inverse Theorem and the Inverse Theorem for epr-Sequences, $\operatorname{apr}\left(C^{-1}\right)=a_{p+1}^{\prime} \mathrm{N} \cdots \mathrm{A} a_{k-1}^{\prime} \cdots a_{2}^{\prime} a_{1}^{\prime}$ and $\operatorname{epr}\left(C^{-1}\right)=\mathrm{N} \ell_{p}^{\prime} \cdots \ell_{2}^{\prime} \ell_{1}^{\prime} \mathrm{A}$, which contradicts Lemma 2.6 .

\section{Sequences not containing an A}

In this section, we confine our attention to the apr-sequences not containing $\mathrm{A}$ as a subsequence, for which a complete characterization will be provided (see Theorem [3.6). This characterization is then used to obtain a necessary condition for a sequence to be the apr-sequence of a symmetric matrix over an arbitrary field $\mathbb{F}$. We will start by focusing on sequences that begin with SN. We introduce two matrices that are central to this section:

$$
L_{2}(a):=\left[\begin{array}{ll}
1 & 1 \\
1 & a
\end{array}\right] \text { and } A\left(K_{2}\right):=\left[\begin{array}{ll}
0 & 1 \\
1 & 0
\end{array}\right]
$$

where $a \in \mathbb{F}$.

Lemma 3.1. Let $B \in \mathbb{F}^{n \times n}$ and $n \geq 3$. Suppose that $\operatorname{apr}(B)=a_{1} a_{2} \cdots a_{n-1}$. Then the following statements hold:

1. If $B=J_{n-k} \oplus O_{k}$ for some integer $k$ with $1 \leq k \leq n-1$, then $\operatorname{apr}(B)=\operatorname{SN} \bar{N}$.

2. If $B=L_{2}(a) \oplus O_{n-2}$ for some $a \in \mathbb{F}$, then $\operatorname{apr}(B)=\operatorname{SN} \bar{N}$.

3. If $B=A\left(K_{2}\right) \oplus A\left(K_{2}\right) \oplus \cdots \oplus A\left(K_{2}\right) \oplus O_{k}$ for some integer $k$ with $0 \leq k \leq n-2$, then $\operatorname{apr}(B)=\operatorname{SiNS} \overline{\mathrm{N}}$, with $\overline{\mathrm{N}}$ containing $k$ copies of $\mathrm{N}$. 
Proof. The verification of Statements (1) and (2) is omitted, since it is trivial. Statement (3) is established by examining two cases. First, consider the case when $B=A\left(K_{2}\right) \oplus O_{k}$ with $k \geq 1$. In that case, obviously, $a_{1}=\mathrm{S}$, and, since every almost-principal submatrix of $B$ of order 2 or larger would contain a zero row or a zero column, we would have $\operatorname{apr}(B)=\operatorname{SN} \bar{N}$.

Finally, to establish the remaining cases of Statements (3), by Observation 1.15, it suffices to show that the matrix

$$
C=A\left(K_{2}\right) \oplus A\left(K_{2}\right) \oplus \cdots \oplus A\left(K_{2}\right),
$$

with at least two copies of $A\left(K_{2}\right)$, has apr-sequence Sns $\overline{N S}$. Let $\operatorname{apr}(C)=a_{1}^{\prime} a_{2}^{\prime} \cdots a_{m-1}^{\prime}$, where $m \geq 4$ (thus, $C$ is an $m \times m$ matrix and $m$ is even). Clearly, $a_{1}^{\prime}=\mathrm{S}$. Let $p$ be an odd integer with $3 \leq p \leq m-1$. We now show that $a_{p}^{\prime}=\mathrm{S}$. Notice that

$$
B[\{1,2, \ldots, p\},\{1,2, \ldots, p+1\} \backslash\{p\}]=A\left(K_{2}\right) \oplus A\left(K_{2}\right) \oplus \cdots \oplus A\left(K_{2}\right) \oplus J_{1}
$$

(with $\frac{p-1}{2}$ copies of $A\left(K_{2}\right)$ ), which is nonsingular, and that $B[\{1,2, \ldots, p\},\{2,3, \ldots, p+1\}]$ is singular, since its second row consists entirely of zeros (i.e., it is a zero row). Hence, $a_{p}^{\prime}=\mathrm{S}$, as desired.

We now show that $a_{q}^{\prime}=\mathrm{N}$ if $q$ is even. First, observe that any principal submatrix of $B$ of odd order contains a zero row and a zero column. Let $q$ be an even integer with $2 \leq q \leq m-2$, and suppose that $B[\alpha \cup\{i\}, \alpha \cup\{j\}]$ is a $q \times q$ almost-principal submatrix; thus, $i \neq j$ and $|\alpha|=q-1$. Hence, $B[\alpha \cup\{i, j\}]$ is a $(q+1) \times(q+1)$ (principal) submatrix of odd order, implying that $B[\alpha \cup\{i, j\}]$ contains a zero row and a zero column. Hence, any $q \times q$ almost-principal submatrix of $B[\alpha \cup\{i, j\}]$ contains either a zero row or a zero column. Then, as $B[\alpha \cup\{i\}, \alpha \cup\{j\}]$ is a submatrix of $B[\alpha \cup\{i, j\}], B[\alpha \cup\{i\}, \alpha \cup\{j\}]$ is singular. It follows that $a_{q}^{\prime}=\mathrm{N}$. We conclude that $\operatorname{apr}(C)=$ SNSTS, as desired.

Proposition 3.2. Let $B \in \mathbb{F}^{n \times n}$ be symmetric. Suppose that $\operatorname{apr}(B)=\operatorname{SN} a_{3} a_{4} a_{5} \cdots a_{n-1}$. Then one of the following statements holds:

1. There exists a generalized permutation matrix $P$ and a nonzero constant $c \in \mathbb{F}$ such that $c P^{T} B P=J_{n-k} \oplus O_{k}$ for some integer $k$ with $1 \leq k \leq n-1$. Moreover, $\operatorname{apr}(B)=\operatorname{SN} \bar{N}$.

2. There exists a generalized permutation matrix $P$, a nonzero constant $c \in \mathbb{F}$ and $a \in \mathbb{F}$ such that $c P^{T} B P=L_{2}(a) \oplus O_{n-2}$. Moreover, $\operatorname{apr}(B)=\operatorname{SN} \overline{\mathrm{N}}$.

3. There exists a generalized permutation matrix $P$ such that $P^{T} B P=A\left(K_{2}\right) \oplus A\left(K_{2}\right) \oplus$ $\cdots \oplus A\left(K_{2}\right) \oplus O_{k}$ for some integer $k$ with $0 \leq k \leq n-2$. Moreover, $\operatorname{apr}(B)=\mathrm{S} \overline{\mathrm{NS}} \overline{\mathrm{N}}$, with $\overline{\mathrm{N}}$ containing $k$ copies of $\mathrm{N}$.

Proof. Suppose that $B=\left[b_{i j}\right]$. Since apr $(B)$ begins with $\mathrm{S}, B$ contains at least one nonzero off-diagonal entry. It suffices to show that the first sentence of one of Statements (1), (2) and (3) holds, since the remaining part of the statements follows immediately from Lemma 3.1. We proceed by examining two cases.

Case 1: $B$ contains a row with more than one nonzero off-diagonal entry. 
We now show that Statement (1) holds. Since a simultaneous permutation of the rows and columns of $B$ leaves $\operatorname{apr}(B)$ invariant, we may assume that the first row of $B$ contains more than one nonzero off-diagonal entry. Furthermore, we may assume that $b_{1 j} \neq 0$ for $j \in\{2,3, \ldots, p\}$ for some $p \geq 3$, and that $b_{1 j}=b_{j 1}=0$ for $j \in\{p+1, p+2, \ldots, n\}$ (note that $\{p+1, p+2, \ldots, n\}$ is empty if $n=3)$. Let $\alpha=\{2,3, \ldots, p\}$ and $\beta=\{p+1, p+2, \ldots n\}$. Since $a_{2}=\mathrm{N}, b_{11} b_{23}-b_{12} b_{13}=\operatorname{det} B[\{1,2\},\{1,3\}]=0$. Then, as $b_{12} b_{13} \neq 0, b_{11} \neq 0$. Since multiplying $B$ by a nonzero constant leaves $\operatorname{apr}(B)$ invariant, we may assume that $b_{11}=1$. Furthermore, we may assume that $b_{1 j}=b_{j 1}=1$ for all $j \in \alpha$, as multiplying a row and column of $B$ by a nonzero constant leaves $\operatorname{apr}(B)$ invariant.

Thus far, we have that $b_{11}=1$, that $b_{1 j}=b_{j 1}=1$ for all $j \in \alpha$, and that $b_{1 j}=b_{j 1}=0$ for all $j \in \beta$. Now, note that for all $i, j \in\{1,2, \ldots, n\}$, with $i \neq j$, and all $t \in\{1,2, \ldots, n\} \backslash\{i, j\}$,

$$
\operatorname{det} B[\{t, i\},\{t, j\}]=b_{t t} b_{i j}-b_{t i} b_{t j}
$$

Since $a_{2}=\mathrm{N}$, we have that, for $i \neq j$ and $t \in\{1,2, \ldots, n\} \backslash\{i, j\}$,

$$
b_{t t} b_{i j}=b_{t i} b_{t j}
$$

Since $b_{11}=1, b_{i j}=b_{1 i} b_{1 j}$ for all $i, j \in\{2,3, \ldots, n\}$ with $i \neq j$. Thus, if $i, j \in \alpha$ and $i \neq j$, then $b_{i j}=1$, implying that every off-diagonal entry of $B[\alpha]$ is 1 . Moreover, if $i \in \beta$ or $j \in \beta$, with $i \neq j$, then $b_{i j}=0$. Hence, $B[\beta]$ is a diagonal matrix and $B=B[\alpha] \oplus B[\beta]$.

We now show that $B[\alpha]=J_{p}$. Observe that if $t \in \alpha$ and $i, j \in\{1,2, \ldots, p\} \backslash\{t\}$ with $i \neq j$, then $b_{t t}=b_{t i} b_{t j} / b_{i j}=1$. Hence, $B[\alpha]=J_{p}$.

We now show that $B[\beta]=O_{n-p}$. Since $a_{1}=\mathrm{S}$, it follows that $n>p$, as otherwise we would have $n=p$, which would imply that $B=B[\alpha]=J_{p}$, whose apr-sequence is AN $\overline{\mathrm{N}}$, which is a contradiction. It follows that $\beta$ is nonempty. If $t \in \beta$, then $b_{t t}=b_{t 1} b_{t 2} / b_{12}=0$. Thus, $B[\beta]=O_{n-p}$. Then, with $k:=n-p$, we have that $B=J_{n-k} \oplus O_{k}$. It is easy to see that the operations performed on $B$ that resulted in the matrix $J_{n-k} \oplus O_{k}$ is accomplished by finding an appropriate generalized permutation matrix $P$ and a nonzero constant $c$ such that $c P^{T} B P=J_{n-k} \oplus O_{k}$. Moreover, observe that $1 \leq k \leq n-3 \leq n-1$. Hence, Statement (1) holds.

Case 2: Each row of $B$ contains at most one nonzero off-diagonal entry.

Since a simultaneous permutation of the rows and columns of $B$ leaves $\operatorname{apr}(B)$ invariant, we may assume that $b_{12} \neq 0$ and $b_{1 j}=0$ for $j \in\{2,3, \ldots, n\}$. Since $B$ does not contain a row with more than one nonzero off-diagonal entry, and because $B$ is symmetric, $B=$ $B[\{1,2\}] \oplus B[\{3,4, \ldots, n\}]$. Moreover, since multiplying a row and column of $B$ by a nonzero constant leaves $\operatorname{apr}(B)$ invariant, we may assume that $b_{12}=b_{21}=1$. Then, as $a_{2}=\mathrm{N}$, $0=\operatorname{det} B[\{1, j\},\{2, j\}]=b_{j j}$ for $j \in\{3,4, \ldots, n\}$. Hence, $B[\{3,4, \ldots, n\}]$ has zero diagonal. Subcase A: $B[\{3,4, \ldots, n\}]=O_{n-2}$.

If $b_{11}=b_{22}=0$, then $B=A\left(K_{2}\right) \oplus O_{n-2}$, implying that Statement (3) holds. Now, suppose that $b_{11} \neq 0$ or $b_{22} \neq 0$. We may assume that $b_{11} \neq 0$. Then by multiplying $B$ by $\frac{1}{b_{11}}$, and then multiplying row 2 and column 2 of $B$ by $b_{11}$, we obtain the matrix $L_{2}(a) \oplus O_{n-2}$ for some a. Without loss of generality, we may assume that $B=L_{2}(a) \oplus O_{n-2}$. It is easy to verify that the operations performed on $B$ that led to the matrix $L_{2}(a) \oplus O_{n-2}$ is accomplished 
by finding an appropriate generalized permutation matrix $P$ and a nonzero constant $c$ such that $c P^{T} B P=L_{2}(a) \oplus O_{n-2}$. Hence, Statement (2) holds.

Subcase B: $B[\{3,4, \ldots, n\}] \neq O_{n-2}$.

Since $B[\{3,4, \ldots, n\}]$ has zero diagonal, $B[\{3,4, \ldots, n\}]$ must have a nonzero off-diagonal entry. Without loss of generality, we may assume that $b_{34} \neq 0$ and $b_{3 j}=0$ for $j \in\{4,5, \ldots n\}$. Then, as $a_{2}=\mathrm{N}, 0=\operatorname{det} B[\{1,3\},\{1,4\}]=b_{11} b_{34}$ and $0=\operatorname{det} B[\{2,3\},\{2,4\}]=b_{22} b_{34}$. Since $b_{34} \neq 0, b_{11}=b_{22}=0$. Hence, $B=A\left(K_{2}\right) \oplus B[\{3,4, \ldots, n\}]$. Since every almostprincipal minor of $B[\{3,4, \ldots, n\}]$ is an almost-principal minor of $B, \operatorname{apr}(B[\{3,4, \ldots, n\}])$ begins with SN. By our assumption in the present case (Case 2), $B[\{3,4, \ldots, n\}]$ does not contain a row with more than one nonzero off-diagonal entry. Thus, we can apply our findings in Subcase A and Subcase B of the present case (Case 2) to the matrix $B[\{3,4, \ldots, n\}]$ : Since $B[\{3,4, \ldots, n\}]$ has zero diagonal, we conclude that we may assume that either $B[\{3,4, \ldots, n\}]=A\left(K_{2}\right) \oplus B[\{5,6, \ldots, n\}]$ (if $\left.n \geq 5\right)$ or $B[\{3,4, \ldots, n\}]=A\left(K_{2}\right)$ (if $n=4)$. Hence, we must have $B=A\left(K_{2}\right) \oplus A\left(K_{2}\right)$ if $n=4$, and $B=A\left(K_{2}\right) \oplus A\left(K_{2}\right) \oplus$ $B[\{5,6, \ldots, n\}]$ if $n \geq 5$. It is not hard to see now that continuing this process will allow us to assume, without loss of generality, that $B=A\left(K_{2}\right) \oplus A\left(K_{2}\right) \oplus \cdots \oplus A\left(K_{2}\right) \oplus O_{k}$ for some integer $k$ with $0 \leq k \leq n-2$ (where the parity of $k$ is the same as that of $n$ ); hence, Statement (3) holds.

We now turn our attention to sequences that begin with SS.

Proposition 3.3. A sequence of the form $\mathrm{SS} a_{3} a_{4} \cdots a_{n-3} \mathrm{NS}$ is not the apr-sequence of $a$ symmetric matrix over $\mathbb{F}$.

Proof. Suppose to the contrary that there exists a symmetric matrix $B \in \mathbb{F}^{n \times n}$ such that $\operatorname{apr}(B)=\mathbf{S S} a_{3} a_{4} \cdots a_{n-3}$ NS. Observe that $B$ is singular (otherwise, the Inverse Theorem would imply that $B^{-1}$ has apr-sequence $\mathrm{SN} a_{n-3} \cdots a_{4} a_{3} \mathrm{SS}$, which would contradict Proposition 3.2). Hence, $\operatorname{rank}(B) \leq n-1$. Since $\operatorname{apr}(B)$ ends with $\mathrm{S}, B$ contains a nonsingular $(n-1) \times(n-1)$ (almost-principal) submatrix, implying that $\operatorname{rank}(B)=n-1$. Since the rank of $B$ is principal, $B$ contains a nonsingular $(n-1) \times(n-1)$ principal submatrix, say, $B^{\prime}$. Without loss of generality, we may assume that $B^{\prime}=B[\{1,2, \ldots, n-1\}]$. By the Inheritance Theorem, $\operatorname{apr}\left(B^{\prime}\right)$ ends with $\mathrm{N}$. Then, as $B^{\prime}$ is nonsingular, $\left(B^{\prime}\right)^{-1}$ is a diagonal matrix, implying that $B^{\prime}$ is also a diagonal matrix. Then, as $B^{\prime}$ is nonsingular, $b_{j j} \neq 0$ for all $j \in\{1,2, \ldots, n-1\}$. Since $\operatorname{apr}(B)$ begins with $\mathrm{S}$, the last row (and last column) of $B$ must contain a nonzero off-diagonal entry. Without loss of generality, we may assume that $b_{1 n} \neq 0$. Now, note that

$$
\operatorname{det}(B[\{1,2, \ldots, n-2\},\{1,2, \ldots, n\} \backslash\{1, n-1\}])=(-1)^{n-1} b_{1 n} \prod_{j=2}^{n-2} b_{j j} \neq 0
$$

Hence, $B$ contains an $(n-2) \times(n-2)$ nonsingular almost-principal submatrix, which contradicts the fact that $\operatorname{apr}(B)=\mathrm{SS} a_{3} a_{4} \cdots a_{n-3}$ NS. 
Lemma 3.4. Let $B \in \mathbb{F}^{n \times n}$ be symmetric, and let $k$ be an even integer. Suppose that $\operatorname{apr}(B)=a_{1} a_{2} \cdots a_{n-1}$ and $a_{k} a_{k+1}=$ NS. Let $B^{\prime}$ be $a(k+2) \times(k+2)$ principal submatrix of B. If $B^{\prime}=A\left(K_{2}\right) \oplus A\left(K_{2}\right) \oplus \cdots \oplus A\left(K_{2}\right)$, then $a_{1} a_{2}=\mathrm{SN}$.

Proof. Suppose that $B^{\prime}=A\left(K_{2}\right) \oplus A\left(K_{2}\right) \oplus \cdots \oplus A\left(K_{2}\right)$. Thus, $a_{1}=\mathrm{S}$. If $k=2$, then there is nothing to prove, and if $n=k+2$, then the desired conclusion follows from Lemma 3.1; thus, we assume that $k \geq 4$, and that $n \geq k+3$. Without loss of generality, we may assume that $B^{\prime}=B[\{1,2, \ldots, k+2\}]$. We now show that $B[\{k+3, k+4, \ldots, n\}]$ has zero diagonal, and that

$$
B=B^{\prime} \oplus B[\{k+3, k+4, \ldots, n\}] .
$$

Suppose that $B=\left[b_{i j}\right]$. To see that $B[\{k+3, k+4, \ldots, n\}]$ has zero diagonal, let $j \in$ $\{k+3, k+4, \ldots, n\}$ and $\alpha=\{1,2, \ldots, k\} \cup\{j\}$. Then, as $a_{k}=\mathrm{N}$,

$$
0=\operatorname{det} B[\alpha \backslash\{1\}, \alpha \backslash\{2\}]=(-1)^{\frac{k-2}{2}} b_{j j} \operatorname{det}(M),
$$

where $M$ is the $(k-1) \times(k-1)$ matrix

$$
M=J_{1} \oplus A\left(K_{2}\right) \oplus A\left(K_{2}\right) \oplus \cdots \oplus A\left(K_{2}\right) .
$$

Then, as $\operatorname{det}(M) \neq 0, b_{j j}=0$. It follows that $B[\{k+3, k+4, \ldots, n\}]$ has zero diagonal, as desired.

Now, to show that

$$
B=B^{\prime} \oplus B[\{k+3, k+4, \ldots, n\}],
$$

let $p, q \in\{1,2, \ldots, k+2\}$ and $j \in\{k+3, k+4, \ldots, n\}$, where $p$ is odd and $q$ is even. We now show that $b_{p j}=0$ and $b_{q j}=0$.

Case 1: $p \leq k-2$ and $q \leq k-2$.

Let $\alpha=\{1,2, \ldots, k\}$. It is easy to see that

$$
\operatorname{det} B[\alpha,(\alpha \backslash\{p+1\}) \cup\{j\}]=(-1)^{p+k} b_{p j} \operatorname{det}(F)
$$

and

$$
\operatorname{det} B[\alpha,(\alpha \backslash\{q-1\}) \cup\{j\}]=(-1)^{q+k} b_{q j} \operatorname{det}(G),
$$

for some matrices $F$ and $G$ that are permutationally similar to the $(k-1) \times(k-1)$ matrix

$$
J_{1} \oplus A\left(K_{2}\right) \oplus A\left(K_{2}\right) \oplus \cdots \oplus A\left(K_{2}\right) .
$$

Since $a_{k}=\mathrm{N}$, $\operatorname{det} B[\alpha,(\alpha \backslash\{p+1\}) \cup\{j\}]=0$ and $\operatorname{det} B[\alpha,(\alpha \backslash\{q-1\}) \cup\{j\}]=0$. Then, as $F$ and $G$ are nonsingular, $b_{p j}=0$ and $b_{q j}=0$, as desired.

Case 2: $p>k-2$ and $q>k-2$.

Let $\beta=\{1,2, \ldots, k-2\}$. It is easy to see that

$$
\operatorname{det} B[\beta \cup\{p, p+1\}, \beta \cup\{p, j\}]=-b_{p j} \operatorname{det}(H)
$$

and

$$
\operatorname{det} B[\beta \cup\{q-1, q\}, \beta \cup\{q, j\}]=b_{q j} \operatorname{det}(H),
$$


where $H$ is the $(k-1) \times(k-1)$ matrix

$$
H=A\left(K_{2}\right) \oplus A\left(K_{2}\right) \oplus \cdots \oplus A\left(K_{2}\right) \oplus J_{1} .
$$

Since $a_{k}=\mathrm{N}$, det $B[\beta \cup\{p, p+1\}, \beta \cup\{p, j\}]=0$ and $\operatorname{det} B[\beta \cup\{q-1, q\}, \beta \cup\{q, j\}]=0$. Then, as $H$ is nonsingular, $b_{p j}=0$ and $b_{q j}=0$, as desired.

It follows from Case 1 and Case 2 that

$$
B=B^{\prime} \oplus B[\{k+3, k+4, \ldots, n\}],
$$

as desired.

To conclude the proof, we show that $a_{2}=\mathrm{N}$. If $B[\{k+3, k+4, \ldots, n\}]$ is the zero matrix, then the desired conclusion follows from Lemma 3.1. Thus, assume that $B[\{k+$ $3, k+4, \ldots, n\}]$ is not the zero matrix. Since $B^{\prime}=A\left(K_{2}\right) \oplus A\left(K_{2}\right) \oplus \cdots \oplus A\left(K_{2}\right)$, it suffices to show that there exists a generalized permutation matrix $P$ such that

$$
P^{T} B[\{k+3, k+4, \ldots, n\}] P=A\left(K_{2}\right) \oplus A\left(K_{2}\right) \oplus \cdots \oplus A\left(K_{2}\right) \oplus O_{t}
$$

for some integer $t$ with $t \geq 0$, since that would imply that there exists a generalized permutation matrix $Q$ such that

$$
Q^{T} B Q=A\left(K_{2}\right) \oplus A\left(K_{2}\right) \oplus \cdots \oplus A\left(K_{2}\right) \oplus O_{t}
$$

and, therefore, that $a_{2}=\mathrm{N}$ (see Lemma 3.1). Since $B[\{k+3, k+4, \ldots, n\}]$ is a nonzero matrix with zero diagonal, $n \geq k+4$. If $n=k+4$, then the fact that $B[\{k+3, k+4, \ldots, n\}]$ has zero diagonal immediately implies that there exists a generalized permutation matrix $P$ such that $P^{T} B[\{k+3, k+4, \ldots, n\}] P=A\left(K_{2}\right)$, as desired. Thus, we assume that $n \geq k+5$ (implying that the order of $B[\{k+3, k+4, \ldots, n\}]$ is greater than or equal to 3$)$. Since $B[\{k+3, k+4, \ldots, n\}]$ has zero diagonal, Proposition 3.2 implies that it suffices to show that $\operatorname{apr}(B[\{k+3, k+4, \ldots, n\}])$ begins with SN. We start by showing that $\operatorname{apr}(B[\{k+3, k+4, \ldots, n\}])$ begins with $\mathrm{S}$. Since $B[\{k+3, k+4, \ldots, n\}]$ is a nonzero matrix with zero diagonal, $\operatorname{apr}(B[\{k+3, k+4, \ldots, n\}])$ does not begin with $N$. Suppose to the contrary that $\operatorname{apr}(B[\{k+3, k+4, \ldots, n\}])$ begins with $A$. Since $B[\{k+3, k+4, \ldots, n\}]$ has zero diagonal, $B[\{k+3, k+4, \ldots, n\}]=J_{n-k-2}-I_{n-k-2}$. Let $\theta=\{1,2, \ldots, k-2\}$. Note that $B[\theta]=A\left(K_{2}\right) \oplus A\left(K_{2}\right) \oplus \cdots \oplus A\left(K_{2}\right)$, and that

$$
B[\theta \cup\{k+3, k+4\}, \theta \cup\{k+3, k+5\}]=B[\theta] \oplus B[\{k+3, k+4\},\{k+3, k+5\}] .
$$

Then, as

$$
B\left[\{k+3, k+4\},\{k+3, k+5\}=\left[\begin{array}{ll}
0 & 1 \\
1 & 1
\end{array}\right]\right.
$$

is nonsingular, $B[\theta] \oplus B[\{k+3, k+4\},\{k+3, k+5\}]$ is nonsingular, implying that $B[\theta \cup$ $\{k+3, k+4\}, \theta \cup\{k+3, k+5\}]$ is nonsingular, a contradiction to the fact that $a_{k}=\mathrm{N}$. Hence, it follows that $\operatorname{apr}(B[\{k+3, k+4, \ldots, n\}])$ begins with $\mathbf{S}$. 
It now remains to show that the second letter in $\operatorname{apr}(B[\{k+3, k+4, \ldots, n\}])$ is $\mathrm{N}$. Let $p, q, r \in\{k+3, k+4, \ldots, n\}$ be distinct integers, and let $\mu=\{1,2, \ldots, k+2\}$. We now show that $\operatorname{det}(B[\{p, q\},\{p, r\}])=0$. Notice that $B[\mu]=B^{\prime}$, and that

$$
B[\mu \cup\{p, q\}, \mu \cup\{p, r\}]=B^{\prime} \oplus B[\{p, q\},\{p, r\}] .
$$

Since $a_{k}=\mathrm{N}, B[\mu \cup\{p, q\}, \mu \cup\{p, r\}]$ is singular. Then, as $B^{\prime}$ is nonsingular, $B[\{p, q\},\{p, r\}]$ is singular, implying that $\operatorname{det}(B[\{p, q\},\{p, r\}])=0$, as desired. Hence, every $2 \times 2$ almostprincipal submatrix of $B[\{k+3, k+4, \ldots, n\}]$ is singular, implying that $\operatorname{apr}(B[\{k+3, k+$ $4, \ldots, n\}])$ begins with $\mathrm{SN}$, as desired.

Theorem 3.5. Let $B \in \mathbb{F}^{n \times n}$ be symmetric. Suppose that $\operatorname{apr}(B)=a_{1} a_{2} \cdots a_{n-1}$ and $a_{k} a_{k+1}=\mathrm{NS}$ for some $k$. Then $a_{1} a_{2}=\mathrm{SN}$.

Proof. Suppose to the contrary that $a_{1} a_{2} \neq$ SN. Since $\operatorname{apr}(B)$ contains NS, Observation 2.1 implies that $a_{1} \neq \mathrm{N}$, and Theorem 2.8 implies that $a_{1} a_{2}$ does not contain an A. It follows that $a_{1} a_{2}=\mathrm{SS}$. Since $a_{k+1}=\mathrm{S}, B$ contains a nonsingular $(k+1) \times(k+1)$ almost-principal submatrix, say, $B[\alpha \cup\{i\}, \alpha \cup\{j\}]$ (thus, $i \neq j$ and $|\alpha|=k$ ). Let $B^{\prime}=B[\alpha \cup\{i, j\}]$ and $\operatorname{apr}\left(B^{\prime}\right)=a_{1}^{\prime} a_{2}^{\prime} \cdots a_{k+1}^{\prime}$. Since $a_{k}=\mathrm{N}$, the Inheritance Theorem implies that $a_{k}^{\prime}=\mathrm{N}$. Since $B^{\prime}$ contains the nonsingular $(k+1) \times(k+1)$ almost-principal submatrix $B[\alpha \cup\{i\}, \alpha \cup\{j\}]$ as a submatrix, $a_{k+1}^{\prime} \in\{\mathrm{A}, \mathrm{S}\}$. Thus, $a_{k}^{\prime} a_{k+1}^{\prime} \in\{\mathrm{NA}, \mathrm{NS}\}$. By Theorem 2.7, $a_{k}^{\prime} a_{k+1}^{\prime}=\mathrm{NS}$. Thus, $\operatorname{apr}\left(B^{\prime}\right)=a_{1}^{\prime} a_{2}^{\prime} \cdots a_{k-1}^{\prime}$ NS. Since $\operatorname{apr}\left(B^{\prime}\right)$ contains NS, Observation 2.1 implies that $a_{1}^{\prime} \neq \mathrm{N}$, Theorem 2.8 implies that $a_{1}^{\prime} a_{2}^{\prime}$ does not contain an A, and Proposition 3.3 implies that $a_{1}^{\prime} a_{2}^{\prime} \neq \mathrm{SS}$. Thus, $a_{1}^{\prime} a_{2}^{\prime}=\mathrm{SN}$, and, therefore, $\operatorname{apr}\left(B^{\prime}\right)=\mathrm{SN} a_{3}^{\prime} a_{4}^{\prime} \cdots a_{k-1}^{\prime}$ NS. It follows from Proposition 3.2 that there exists a generalized permutation matrix $P$ such that

$$
P^{T} B^{\prime} P=A\left(K_{2}\right) \oplus A\left(K_{2}\right) \oplus \cdots \oplus A\left(K_{2}\right),
$$

and that $k$ is even. Without loss of generality, we may assume that

$$
B^{\prime}=A\left(K_{2}\right) \oplus A\left(K_{2}\right) \oplus \cdots \oplus A\left(K_{2}\right) .
$$

By Lemma 3.4, $a_{1} a_{2}=\mathrm{SN}$, a contradiction to the fact that $a_{1} a_{2}=\mathrm{SS}$.

The sequences not containing an A that are realized as the apr-sequence of a symmetric matrix over a field $\mathbb{F}$ are characterized:

Theorem 3.6. Let $a_{1} a_{2} \cdots a_{n-1}$ be a sequence from $\{\mathrm{S}, \mathrm{N}\}$ and $\mathbb{F}$ be a field. Then $a_{1} a_{2} \cdots a_{n-1}$ is the apr-sequence of a symmetric matrix $B \in \mathbb{F}^{n \times n}$ if and only if $a_{1} a_{2} \cdots a_{n-1}$ has one of the following forms:

1. $N \overline{\mathrm{N}}$.

2. $\mathrm{SN} \overline{\mathrm{N}}$.

3. $\operatorname{SNS} \overline{\mathrm{NS}} \overline{\mathrm{N}}$. 


\section{4. $\operatorname{SS} \overline{\mathrm{S}} \overline{\mathrm{N}}$.}

Proof. Let $\sigma=a_{1} a_{2} \cdots a_{n-1}$. Suppose that $\sigma$ is the apr-sequence of some symmetric matrix $B \in \mathbb{F}^{n \times n}$. If $a_{1}=\mathrm{N}$, then $\sigma$ has form (1) (see Observation 2.1). Thus, assume that $a_{1}=\mathrm{S}$. Since the apr-sequence $\mathrm{S}$ is not attainable (by a $2 \times 2$ matrix), $n \geq 3$. If $a_{2}=\mathrm{N}$, then Proposition 3.2 implies that $\sigma$ must have one of the forms (2) or (3). Finally, assume that $a_{2}=\mathrm{S}$. Then, by Theorem 3.5, $\sigma$ does not contain NS. It follows that $\sigma$ must have form (4).

For the other direction, suppose that $\sigma$ has one of the forms (1)-(4). If $\sigma=N \bar{N}$, then $\operatorname{apr}\left(O_{n}\right)=\sigma$. If $\sigma$ has the form (2) or (3), then the desired conclusion follows from Lemma 3.1. Finally, suppose that $\sigma$ has the form (4); thus, $n \geq 3$. Because of Observation 1.15, it suffices to reach the desired conclusion in the case when $\sigma$ does not contain an $\mathrm{N}$; thus, we assume that $\sigma=\mathrm{SS} \overline{\mathrm{S}}$. Let $B^{\prime}=J_{2} \oplus I_{n-2}$ and $\operatorname{apr}\left(B^{\prime}\right)=a_{1}^{\prime} a_{2}^{\prime} \ldots a_{n-1}^{\prime}$. We now show that $\operatorname{apr}\left(B^{\prime}\right)=\sigma$. It is obvious that $a_{1}^{\prime}=\mathrm{S}$. Let $k \in\{2,3, \ldots, n-1\}$ and $\alpha=\{1,2, \ldots, k+1\}$. Now, observe that $C[\alpha \backslash\{1\}, \alpha \backslash\{k+1\}]$ has a zero row, and that $C[\alpha \backslash\{1\}, \alpha \backslash\{2\}]=I_{k}$. Hence, $C$ contains both a singular and a nonsingular $k \times k$ almost-principal submatrix, implying that $\operatorname{apr}(C)=\operatorname{SS} \overline{\mathrm{S}}=\sigma$, as desired.

Combining Theorem 3.6 with Theorem 2.8 leads to a necessary condition for a sequence to be the apr-sequence of a symmetric matrix over an arbitrary field $\mathbb{F}$ :

Theorem 3.7. Let $\mathbb{F}$ be a field. Let $n \geq 3$ and $\sigma=a_{1} a_{2} \cdots a_{n-1}$ be a sequence from $\{\mathrm{A}, \mathrm{N}, \mathrm{S}\}$. If $\sigma$ is the apr-sequence of a symmetric matrix $B \in \mathbb{F}^{n \times n}$, then one of the following statements holds:

1. $\sigma=\operatorname{SNS} \overline{\mathrm{NS}} \overline{\mathrm{N}}$.

2. Neither NA nor NS is a subsequence of $\sigma$.

If $\mathbb{F}$ is the field of order 2 , then the converse of Theorem 3.7 does not hold: An exhaustive inspection reveals that the only sequences starting with A that are realized as the aprsequence of a $4 \times 4$ symmetric matrix over the field of order 2 are AAA, ASS, ASN and ANN (since a simultaneous permutation of the rows and columns of a matrix leaves its apr-sequence invariant, this inspection is reduced to checking a total of five matrices); as this list does not include the sequences AAN, AAS and ASA, the converse of Theorem 3.7 does not hold if $\mathbb{F}$ is the field of order 2 .

For fields of characteristic 0, we suspect that the converse of Theorem 3.7 holds. Since Lemma 3.1 implies that $\sigma=\operatorname{SNS} \overline{\mathrm{NS}} \overline{\mathrm{N}}$ is realized as the apr-sequence of a symmetric matrix (over any field), the converse of Theorem 3.7 holds if the following statement holds: If $\mathbb{F}$ is a field and $\sigma$ is a sequence from $\{\mathrm{A}, \mathrm{N}, \mathrm{S}\}$ of length $n-1$, with $n \geq 3$, that contains neither NA nor NS as a subsequence, then $\sigma$ is the apr-sequence of a symmetric matrix in $\mathbb{F}^{n \times n}$; if $\mathbb{F}$ is a field of characteristic 0, this statement is reminiscent of, and closely related to, Theorem 1.2, which is partly why we speculate that the converse of Theorem 3.7 may hold if $\mathbb{F}$ is a field of characteristic 0 . To establish this statement for fields of characteristic 0 , it would be natural to resort to probabilistic techniques akin to those used in both [5] and [7]. In some instances, these techniques consist of taking an $(n-1) \times(n-1)$ symmetric matrix $B$ with $\operatorname{apr}(B)=a_{1} a_{2} \cdots a_{n-2}$ and verifying the existence of a bordering strategy to produce an $n \times n$ 
symmetric matrix $B^{\prime}$ such that $\operatorname{apr}\left(B^{\prime}\right):=a_{1}^{\prime} a_{2}^{\prime} \cdots a_{n-1}^{\prime}=a_{1} a_{2} \cdots a_{n-2} a_{n-1}^{\prime}$, where $a_{n-1}^{\prime}$ is prescribed. Roughly speaking, the existence of such a $B^{\prime}$ depends upon sufficient available choice of possible vectors in $\mathbb{F}^{n-1}$. If $B$ is an arbitrary $(n-1) \times(n-1)$ symmetric matrix with $\operatorname{epr}(B)=\mathrm{AA} \cdots \mathrm{A}$ (i.e., the sequence each of whose terms is equal to $\mathrm{A}$ ), then applying the aforementioned probabilistic techniques to $B$ yield an $n \times n$ (symmetric) matrix $B^{\prime}$ with

$\operatorname{epr}\left(B^{\prime}\right)=\mathrm{AA} \cdots \mathrm{AN}$ (see [5. Proposition 4.1]). Similarly, if $C$ is an arbitrary $(n-1) \times(n-1)$ symmetric matrix with $\operatorname{qpr}(C)=\mathrm{AA} \cdots \mathrm{A}$, then applying the aforementioned probabilistic techniques to $C$ yield an $n \times n$ (symmetric) matrix $C^{\prime}$ with $\operatorname{qpr}\left(C^{\prime}\right)=\mathrm{AA} \cdots \mathrm{AN}$ (see [7, Lemma 3.1]). The next example shows that something similar does not hold for apr-sequences, i.e., that if $B$ is an arbitrary $(n-1) \times(n-1)$ symmetric matrix with $\operatorname{apr}(B)=\mathrm{AA} \cdots \mathrm{A}$, then applying the aforementioned probabilistic techniques to $B$ need not yield an $n \times n$ (symmetric) matrix $B^{\prime}$ with $\operatorname{apr}\left(B^{\prime}\right)=\mathrm{AA} \cdots \mathrm{AN}$.

Example 3.8. Let

$$
B=\left[\begin{array}{ccc}
-1 & 1 & 1 \\
1 & -1 & 1 \\
1 & 1 & -1
\end{array}\right] \in \mathbb{R}^{3 \times 3} \quad \text { and } \quad B^{\prime}=\left[\begin{array}{cc}
B & \vec{y} \\
\vec{y} & t
\end{array}\right] \in \mathbb{R}^{4 \times 4}
$$

where $\vec{y}$ and $t$ are arbitrary. Suppose that $\vec{y}=\left[y_{1}, y_{2}, y_{3}\right]^{T}$. Observe that $\operatorname{apr}(B)=$ AA. We now show that there is no $\vec{y} \in \mathbb{R}^{3}$ such that $\operatorname{apr}\left(B^{\prime}\right)=$ AAN. Observe that $\operatorname{det}\left(B^{\prime}[\{1,2,3\},\{2,3,4\}]\right)=2\left(y_{2}+y_{3}\right)=-2 \operatorname{det}\left(B^{\prime}[\{2,3\},\{2,4\}]\right)$. It follows that if all of the order-3 almost-principal minors of $B^{\prime}$ are zero, then some order-2 almost-principal minor of $B^{\prime}$ is zero. Thus, there is no $\vec{y} \in \mathbb{R}^{3}$ such that apr $\left(B^{\prime}\right)=$ AAN.

The next example shows that if $B$ is an arbitrary $(n-1) \times(n-1)$ symmetric matrix with $\operatorname{apr}(B)=\mathrm{SS} \cdots \mathrm{S}$ (i.e., the sequence each of whose terms is equal to $\mathbf{S}$ ), then applying the aforementioned probabilistic techniques to $B$ need not yield an $n \times n$ (symmetric) matrix $B^{\prime}$ with $\operatorname{apr}\left(B^{\prime}\right)=$ SS $\cdots$ SA.

Example 3.9. Let

$$
B=\left[\begin{array}{llll}
1 & 1 & 0 & 0 \\
1 & 1 & 1 & 1 \\
0 & 1 & 1 & 1 \\
0 & 1 & 1 & 1
\end{array}\right] \in \mathbb{F}^{4 \times 4} \quad \text { and } \quad B^{\prime}=\left[\begin{array}{cc}
B & \vec{y} \\
\vec{y} & t
\end{array}\right] \in \mathbb{F}^{5 \times 5},
$$

where $\mathbb{F}$ is an arbitrary field and $\vec{y}$ and $t$ are arbitrary. Observe that $\operatorname{apr}(B)=$ SSS. We now show that there is no $\vec{y} \in \mathbb{F}^{4}$ such that $\operatorname{apr}\left(B^{\prime}\right)=$ SSSA. This is readily seen by noting that $\operatorname{det}\left(B^{\prime}[\{1,2,3,4\},\{2,3,4,5\}]\right)=0$ for all $\vec{y} \in \mathbb{F}^{4}$ (two of the columns of $B^{\prime}[\{1,2,3,4\},\{2,3,4,5\}]$ are the same).

\section{The ap-rank of a symmetric matrix}

This section is devoted to studying the ap-rank of a symmetric matrix over an arbitrary field $\mathbb{F}$. We start with basic observations. 
Observation 4.1. Let $n \geq 2$ and $B \in \mathbb{F}^{n \times n}$ be symmetric. Then ap-rank $(B)$ is equal to the index of the last $\mathrm{A}$ or $\mathrm{S}$ in $\operatorname{apr}(B)$.

Observation 4.2. Let $B \in \mathbb{F}^{n \times n}$ be symmetric. Then $\operatorname{ap}-\operatorname{rank}(B) \leq \operatorname{rank}(B)$.

Observation 4.3. Let $B \in \mathbb{F}^{n \times n}$ be symmetric. Then $\operatorname{ap}-\operatorname{rank}(B)=0$ if and only if $B$ is a diagonal matrix.

Since the inverse of a nonsingular non-diagonal matrix is non-diagonal, the following fact is deduced easily from the relationship between a matrix and its adjoint.

Proposition 4.4. Let $B \in \mathbb{F}^{n \times n}$ be symmetric, non-diagonal and non-singular. Then $\operatorname{ap}-\operatorname{rank}(B)=n-1$.

As we saw earlier (in Theorem (1.6), the rank of a symmetric matrix is equal to the order of a largest nonsingular principal submatrix - which led us to call the rank of such a matrix "principal." A natural question one should ask is whether an analogous connection exists between the rank of a symmetric matrix and the order of a largest nonsingular almostprincipal submatrix; that is, is it the case that the rank and ap-rank of a symmetric matrix is the same? Obviously, the answer is negative, since, for example, for a nonzero diagonal matrix $B$, ap-rank $(B)=0$, while $\operatorname{rank}(B)>0$. Moreover, since for an $n \times n$ matrix $B$ we must have $\operatorname{ap}-\operatorname{rank}(B) \leq n-1, \operatorname{ap}-\operatorname{rank}(B) \neq \operatorname{rank}(B)$ if $B$ is nonsingular. But what can we say if $B$ is non-diagonal and singular? After establishing the following two lemmas, we show that if $B$ is symmetric, non-diagonal and singular, and does not contain a zero row, then $\operatorname{ap}-\operatorname{rank}(B)=\operatorname{rank}(B)$.

Lemma 4.5. Let $B \in \mathbb{F}^{n \times n}$ be symmetric and singular. Suppose that $\operatorname{rank}(B)=r$ and $B$ does not contain a zero row. Let $B[\alpha]$ be an $r \times r$ nonsingular (principal) submatrix of $B$. Then there exists $p \in\{1,2, \ldots, n\} \backslash \alpha$ such that $B[\alpha \cup\{p\}]$ does not contain a zero row.

Proof. Without loss of generality, we may assume that $\alpha=\{1,2, \ldots, r\}$. Suppose to the contrary that the matrix $B[\alpha \cup\{p\}]$ contains a zero row for all $p \in\{1,2, \ldots, n\} \backslash \alpha$ (since $B$ is singular, $\{1,2, \ldots, n\} \backslash \alpha$ is nonempty). It follows that $B=B[\alpha] \oplus C$, where $C$ is an $(n-$ $r) \times(n-r)$ matrix with zero diagonal. Now, observe that $\operatorname{rank}(B)=\operatorname{rank}(B[\alpha])+\operatorname{rank}(C)$. Then, as $\operatorname{rank}(B[\alpha])=\operatorname{rank}(B)$, it follows that $\operatorname{rank}(C)=0$, implying that $C=O_{n-r}$, which contradicts the fact that $B$ does not contain a zero row.

Lemma 4.6. Let $B \in \mathbb{F}^{n \times n}$ be symmetric. Suppose that $\operatorname{apr}(B)=a_{1} a_{2} \cdots a_{n-2} \mathrm{~N}$ and $\operatorname{rank}(B)=n-1$. Then $B$ contains a zero row.

Proof. Suppose that $B=\left[b_{i j}\right]$. Since $\operatorname{apr}(B)$ ends with $\mathrm{N}$, the adjoint of $B$ is a diagonal matrix, and is of rank one, $\operatorname{since} \operatorname{rank}(B)=n-1$. Hence it follows that $B e_{i}=0$ for some standard basis vector $e_{i}$. Thus, the $i$ th column (and hence row) of $B$ is zero.

Theorem 4.7. Let $B \in \mathbb{F}^{n \times n}$ be a symmetric, non-diagonal, singular matrix not containing a zero row. Then ap-rank $(B)=\operatorname{rank}(B)$. 
Proof. Since $B$ is a non-diagonal matrix, $n \geq 2$. Let $r=\operatorname{rank}(B)$. Since ap-rank $(B) \leq r$, it suffices to show that $B$ contains a nonsingular $r \times r$ almost-principal submatrix. Since the rank of $B$ is principal, $B$ contains a nonsingular $r \times r$ principal submatrix, say, $B[\alpha]$. By Lemma 4.5, there exists $p \in\{1,2, \ldots, n\} \backslash \alpha$ such that $B^{\prime}:=B[\alpha \cup\{p\}]$ does not contain a zero row. Since $\operatorname{rank}(B)=r$, and because $B^{\prime}$ contains the nonsingular $r \times r$ matrix $B[\alpha]$, $\operatorname{rank}\left(B^{\prime}\right)=r$. Let $\operatorname{apr}\left(B^{\prime}\right)=a_{1}^{\prime} a_{2}^{\prime} \cdots a_{r}^{\prime}$. Since $B^{\prime}$ is a singular $(r+1) \times(r+1)$ (symmetric) matrix with $\operatorname{rank}\left(B^{\prime}\right)=r$, and because $B^{\prime}$ does not contain a zero row, Lemma 4.6 implies that $a_{r}^{\prime} \neq \mathrm{N}$. Hence, $B^{\prime}$ contains a nonsingular, $r \times r$, almost-principal submatrix. Then, as every almost-principal submatrix of $B^{\prime}$ is also an almost-principal submatrix of $B$, the desired conclusion follows.

Although the rank and ap-rank of a symmetric matrix $B$ are not always the same, the rank cannot exceed the ap-rank by more than one if $B$ is a non-diagonal matrix:

Theorem 4.8. Let $B \in \mathbb{F}^{n \times n}$ be symmetric and non-diagonal. Define the parameter $t:=$ $\max \{|\alpha|: B[\alpha]$ does not contain a zero row $\}$, and let $B^{\prime}$ be the $t \times t$ principal submatrix of $B$ not containing a zero row. Then $\operatorname{rank}(B)-1 \leq \operatorname{ap}-\operatorname{rank}(B) \leq \operatorname{rank}(B)$. Moreover, $\operatorname{ap}-\operatorname{rank}(B)=\operatorname{rank}(B)$ if and only if $B^{\prime}$ is singular. Equivalently, $\operatorname{ap}-\operatorname{rank}(B)=\operatorname{rank}(B)-1$ if and only if $B^{\prime}$ is nonsingular.

Proof. Since $B$ is symmetric and non-diagonal, it is immediate that $n \geq 2$ and $t \geq 2$. Without loss of generality, we may assume that $B^{\prime}=B[1,2, \ldots, t]$. Thus, $B=B^{\prime} \oplus O_{n-t}$. Then, $\operatorname{as} \operatorname{rank}(B)=\operatorname{rank}\left(B^{\prime}\right)$ and $\operatorname{ap}-\operatorname{rank}(B)=\operatorname{ap}-\operatorname{rank}\left(B^{\prime}\right)$, it suffices to show that the desired conclusions hold for the case with $B=B^{\prime}$ (that is, the case with $t=n$ ); thus, we assume that $B=B^{\prime}$. If $B$ is nonsingular, then, by Proposition 4.4, ap-rank $(B)=n-1=$ $\operatorname{rank}(B)-1$. If $B$ is singular, then, by Theorem 4.7, $\operatorname{ap}-\operatorname{rank}(B)=\operatorname{rank}(B)$. It follows that $\operatorname{ap}-\operatorname{rank}(B)=\operatorname{rank}(B)-1$ or $\operatorname{ap}-\operatorname{rank}(B)=\operatorname{rank}(B)$, implying that $\operatorname{rank}(B)-1 \leq$ $\operatorname{ap}-\operatorname{rank}(B) \leq \operatorname{rank}(B)$, as desired.

The remaining two statements, and their equivalency, is established easily using the above $\operatorname{arguments}$ in this proof and the fact that $\operatorname{rank}(B)-1 \leq \operatorname{ap}-\operatorname{rank}(B) \leq \operatorname{rank}(B)$.

Although for a given symmetric matrix $B \in \mathbb{F}^{n \times n}$ we must have $0 \leq \operatorname{rank}(B)-\operatorname{apr}(B) \leq 1$ if $B$ is non-diagonal, $\operatorname{rank}(B)-\operatorname{apr}(B)$ can attain any integer value on the closed interval $[0, n]$ if $B$ is a diagonal matrix, since, for example, $\operatorname{rank}(B)-\operatorname{apr}(B)=r$ if $B$ is a diagonal matrix with $\operatorname{rank}(B)=r$.

\section{Concluding remarks}

Given that the only difference between the epr- and apr-sequence is that the former depends on principal minors, while the latter depends on almost-principal minors, it is worthwhile to compare the state of affairs for epr- and apr-sequences. Although the aprsequence was just introduced (in the present paper), we already have a better understanding of this sequence than of the epr-sequence: The epr-sequences of symmetric matrices over the field of order 2 were completely characterized in [13]; however, for any other field, no 
such characterization exists. In Section [3, the sequences not containing any As that are realized as the apr-sequence of a symmetric matrix over an arbitrary field $\mathbb{F}$ were completely characterized. Moreover, in Section 3, a necessary condition for a sequence to be the aprsequence of a symmetric matrix over a field $\mathbb{F}$ was presented. It is clear, then, that our understanding of apr-sequences is already better than that of epr-sequences.

As stated in Section 1, one of our motivations for introducing the ap-rank and aprsequence of a symmetric matrix was answering Question 1.3, which asks if we should attribute the fact that neither NA nor NS can occur as a subsequence of the qpr-sequence of a symmetric matrix $B \in \mathbb{F}^{n \times n}$ entirely to the dependence of qpr-sequences on almost-principal minors. The following remark answers Question 1.3, under the assumption that $n \geq 3$ (the question is trivial when $n \leq 2$ ).

Remark 5.1. Let $n \geq 3, \mathbb{F}$ be a field and $B \in \mathbb{F}^{n \times n}$ be symmetric. Then the fact that neither NA nor NS can occur as a subsequence of $q \operatorname{pr}(B)$ is attributed entirely to the dependence of $\operatorname{qpr}(B)$ on almost-principal minors if and only if $B$ is a non-diagonal matrix for which there does not exist a generalized permutation matrix such that $P^{T} B P=T_{q}^{p}$ when $p \geq 2$, where $T_{q}^{p}$ is the $n \times n$ matrix

$$
T_{q}^{p}:=\underbrace{A\left(K_{2}\right) \oplus A\left(K_{2}\right) \oplus \cdots \oplus A\left(K_{2}\right)}_{p \text { times }} \oplus O_{q} \in \mathbb{F}^{n \times n} .
$$

We now establish the previous statement. First, suppose that $B$ is a diagonal matrix with $\operatorname{rank}(B)=r$. If $B$ is nonsingular, then $\operatorname{epr}(B)=\mathrm{AAA} \overline{\mathrm{A}}$. If $B$ is singular, then $\operatorname{epr}(B)=\overline{\mathrm{S}} \overline{\mathrm{N}}$, with $\mathrm{S}$ occurring $r$ times and $\mathrm{N}$ occurring $n-r$ times. Since $r$ is equal to the index of the last $\mathrm{A}$ or $\mathrm{S}$ in $q \operatorname{pr}(B)$ (see Observation 1.10), neither NA nor NS is a subsequence of $\operatorname{qpr}(B)$, regardless of what $\operatorname{apr}(B)$ is; hence, the fact that neither NA nor NS is a subsequence of $q \operatorname{pr}(B)$ is not attributed entirely to the dependence of $q \operatorname{pr}(B)$ on almost-principal minors.

Now, suppose that $B$ is a non-diagonal matrix for which there exists a generalized permutation matrix $P$ such that $P^{T} B P=T_{q}^{p}$ for some $p \geq 2$. Then either $\operatorname{epr}(B)=$ NSNSNA (if $q=0$ ) or $\operatorname{epr}(B)=\operatorname{NS} \overline{\mathrm{NS}} \overline{\mathrm{N}}$ (if $q \geq 1$ ), with $\overline{\mathrm{N}}$ containing $q$ copies of $\mathrm{N}$. Moreover, $\operatorname{apr}(B)=$ SNSNS $\overline{\mathrm{N}}$, with $\overline{\mathrm{N}}$ containing $q$ copies of $\mathrm{N}$ (see Proposition $[3.2$ ). Then, as $\operatorname{rank}(B)=n-q$, and because $\operatorname{rank}(B)$ is equal to the index of the last $\mathrm{A}$ or $\mathrm{S}$ in $\operatorname{qpr}(B), \operatorname{qpr}(B)=\mathrm{SSS} \overline{\mathrm{S}} \overline{\mathrm{N}}$. It is easy to see that the fact that neither NA nor NS is a subsequence of $\operatorname{qpr}(B)$ is attributed to both the principal and the almost-principal minors of $B$.

Finally, suppose that $B$ is a non-diagonal matrix for which there does not exist a generalized permutation matrix such that $P^{T} B P=T_{q}^{p}$ when $p \geq 2$. Let $q \operatorname{pr}(B)=q_{1} q_{2} \cdots q_{n}$. It suffices to present an argument based solely on almost-principal minors for the fact that if $q_{k}=\mathrm{N}$ for some $k$, then $q_{j}=\mathrm{N}$ for all $j \geq k$; we present one based on the ap-rank and apr-sequence of $B$ : Suppose that $q_{k}=\mathrm{N}$ for some $k$. Let $\operatorname{apr}(B)=a_{1} a_{2} \cdots a_{n-1}$. If $k=n$, then there is nothing to prove; thus, assume that $k \leq n-1$. Obviously, $a_{k}=\mathrm{N}$. We now show that $\operatorname{apr}(B)$ does not contain NA nor NS as a subsequence. If it was the case that $\operatorname{apr}(B)$ contained NA or NS as a subsequence, then Theorem 3.7 would imply that apr $(B)=\operatorname{SNSNS} \bar{N}$, and, then, Proposition 3.2 would imply that there exists a generalized permutation matrix such that $P^{T} B P=T_{q}^{p}$ for some $p \geq 2$, leading to a contradiction. Hence, neither NA nor 
NS is a subsequence of $\operatorname{apr}(B)$. It follows that $a_{j}=\mathrm{N}$ for all $j \geq k$, and, therefore, that $\operatorname{ap}-\operatorname{rank}(B) \leq k-1$. Then, as $B$ is non-diagonal, Theorem 4.8 implies that $\operatorname{rank}(B) \leq k$. Since $\operatorname{rank}(B)$ is equal to the index of the last A or S in $q \operatorname{pr}(B)$ (see Observation 1.10$), q_{j}=\mathrm{N}$ for all $j \geq k+1$. Then, as $q_{k}=\mathrm{N}$, the desired conclusion follows.

\section{Acknowledgments}

The research of the first author was supported in part by an NSERC Discovery Research Grant RGPIN-2014-06036. The authors express their gratitude to a referee for their careful review, and for many helpful and constructive comments on a previous version which greatly improved the presentation of the paper. Moreover, they thank a referee for bringing Example 3.8 to their attention.

\section{References}

[1] W. Barrett, S. Butler, M. Catral, S. M. Fallat, H. T. Hall, L. Hogben, P. van den Driessche, M. Young. The principal rank characteristic sequence over various fields. Linear Algebra and its Applications 459 (2014), 222-236.

[2] T. Boege, A. D'Alì, T. Kahle, B. Sturmfels. The Geometry of Gaussoids. Foundations of Computational Mathematics, to appear. arXiv:1710.07175.

[3] R. A. Brualdi, L. Deaett, D. D. Olesky, P. van den Driessche. The principal rank characteristic sequence of a real symmetric matrix. Linear Algebra and its Applications 436 (2012), 2137-2155.

[4] R. A. Brualdi, H. Schneider. Determinantal identities: Gauss, Schur, Cauchy, Sylvester, Kronecker, Jacobi, Binet, Laplace, Muir, and Cayley. Linear Algebra and its Applications 52/53 (1983), 769-791.

[5] S. Butler, M. Catral, S. M. Fallat, H. T. Hall, L. Hogben, P. van den Driessche, M. Young. The enhanced principal rank characteristic sequence. Linear Algebra and its Applications 498 (2016), 181-200.

[6] S. Butler, M. Catral, H. T. Hall, L. Hogben, X. Martínez-Rivera, B. Shader, P. van den Driessche. The enhanced principal rank characteristic sequence for Hermitian matrices. Electronic Journal of Linear Algebra 32 (2017), 58-75.

[7] S. M. Fallat, X. Martínez-Rivera. The quasi principal rank characteristic sequence. Linear Algebra and its Applications 548 (2018), 42-56.

[8] S. M. Fallat, D. D. Olesky, P. van den Driessche. The enhanced principal rank characteristic sequence for skew-symmetric matrices. Linear Algebra and its Applications 498 (2016), 366-377. 
[9] C. R. Johnson, J. S. Maybee. Vanishing minor conditions for inverse zero patterns. Linear Algebra and its Applications 178 (1993), 1-15.

[10] C. R. Johnson, R. L. Smith. Inverse M-matrices, II. Linear Algebra and its Applications 435 (2011), 953-983.

[11] R. Kenyon, R. Pemantle. Principal minors and rhombus tilings. Journal of Physics A: Mathematical and Theoretical 47 (2014), 474010.

[12] X. Martínez-Rivera. Classification of families of pr- and epr-sequences. Linear and Multilinear Algebra 65 (2017), 1581-1599.

[13] X. Martínez-Rivera. The enhanced principal rank characteristic sequence over a field of characteristic 2. Electronic Journal of Linear Algebra 32 (2017), 273-290.

[14] X. Martínez-Rivera. The signed enhanced principal rank characteristic sequence. Linear and Multilinear Algebra 66 (2018), 1484-1503.

[15] B. Sturmfels. Open problems in algebraic statistics. In Emerging Applications of Algebraic Geometry, M. Putinar and S. Sullivant, Editors. I.M.A. Volumes in Mathematics and its Applications, 149, Springer, New York, 2009.

[16] B. Sturmfels, E. Tsukerman, L. Williams. Symmetric matrices, Catalan paths, and correlations. Journal of Combinatorial Theory, Series A. 144 (2016), 496-510.

[17] R. A. Willoughby. The inverse M-matrix problem. Linear Algebra and its Applications 18 (1977), 75-94.

[18] F. Zhang (editor). The Schur Complement and its Applications. Springer-Verlag, New York, New York, 2005. 